J. Lake Sci.(湖泊科学), 2009, 21(4): 474-482

http://www.jlakes.org. E-mail: jlakes@niglas.ac.cn

(C2009 by Journal of Lake Sciences

\title{
湖泊沉积物-水界面氧气交换速率的测定及影响因素*
}

\author{
王建军, 沈 吉 ${ }^{* *}$, 张 路, 刘恩峰
}

(中国科学院南京地理与湖泊研究所湖泊与环境国家重点实验室, 南京 210008)

摘 要: 水流启动-停止法可有效获取体积式氧气交换速率 $\left(\mathrm{O}_{2}(t)\right)$, 且该速率与沉积物柱样培养法获得的总氧气交换速率 (TOE)之间具显著相关性; 与由一维溶解氧剖面计算获得的氧气在扩散界面层中的扩散速率和氧气在沉积物中的扩散速率相 比, $\mathrm{O}_{2}(t)$ 与 TOE 不仅能代表氧气扩散速率，而且还包括沉积物中生物呼吸以及生物扰动引起的界面氧气交换速率信息. 此外, 通过比较太湖及南四湖多位点不同沉积物性质条件下界面氧气交换速率, 结果表明界面氧气交换速率在空间尺度上的差异性, 除与生物因素有关外, 还与沉积物有机物质含量显著相关.

关键词：沉积物-水界面; 界面氧气交换; 微溶解氧电极; 太湖; 南四湖

\section{Spatial heterogeneity of oxygen exchange between sediment-water interface in lakes}

\section{WANG Jianjun, SHEN Ji, ZHANG Lu \& LIU Enfeng}

(State Key Laboratory of Lake Science and Environment, Nanjing Institute of Geography and Limnology, Chinese Academy of Sciences, Nanjing 210008, P.R.China)

\begin{abstract}
Volumetric oxygen exchange rate $\left(\mathrm{O}_{2}(t)\right)$, based on measurement by a flow-on/flow-off controlling method, is significantly correlated with TOE (total oxygen exchange) obtained from laboratory-incubated sediment cores. The difference between TOE and DOE (diffusive oxygen exchange in diffusive boundary layers) suggested that there is a fauna-mediated $\mathrm{O}_{2}$ consumption in the two factors. Comparison of $\mathrm{DOE}$ with $\mathrm{DOE}_{\mathrm{s}}$ (diffusive oxygen exchange in sediments) from one-dimensional oxygen concentration profiles at a steady stationary condition, $\mathrm{O}_{2}(t)$ as well as TOE, is an integrated measurement for the diffusion, advection and fauna-mediated $\mathrm{O}_{2}$ consumption. Furthermore, a comparative study of TOE values among sediments from Lake Taihu and Lake Nansihu revealed that the difference from the sites was due to the variation of organic matter concentration in the surface sediments, additionally effects of benthic faunas.
\end{abstract}

Keywords: Sediment-water interface; oxygen exchange; oxygen microsensors; Lake Taihu; Lake Nansihu

研究湖泊沉积物-水界面的氧气生物地球化学循环及随着空间位置的改变而发生的变化, 对沉积物 中其他生物地球化学过程具有基础性的意义, 且也可用来指示人类活动对沉积物及水体的影响 ${ }^{[1]}$. 概括 而言, 沉积物-水界面氧气交换速率(Oxygen Exchange, OE)的测定有两类方法: a, 密闭体系中上覆水氧气 含量的改变. 该方法包括实验室内的沉积物柱样培养法 ${ }^{[2]}$ 和原位箱式法 ${ }^{[3]} ; \mathrm{b}$, 底层扩散边界层氧气含量 剖面. 这包括可以在原位以及实验室内实施的溶氧微电极法 ${ }^{[2,4-7]}$ 以及平面苂光平板法 ${ }^{[8-10]}$. 前者提供沉 积物-水界面总氧气交换速率(Total Oxygen Exchange, TOE), 但该数值不能获得除此之外的更多信息, 如 影响氧气生产或消耗的生物化学过程, 沉积物中氧气的分布以及间隙水氧气动态变化. 而后者能够作为 前者的补充，可以获得沉积物特定深度的氧气分布，通过该方法获得稳态条件下的氧气剖面，可以用来 计算氧气扩散速率(Diffusive Oxygen Exchange, DOE)和沉积物好氧层中体积式氧气交换速率 $\left(\mathrm{O}_{2}(t)\right)^{[2,11]}$.

* 中国科学院知识创新工程重大项目(KZCX1-YW-14)、江苏省自然科学基金(BK2008055)和归国人员基金(翟文川)联合资助. 2008-12-29 收稿; 2009-02-15 收修改稿. 王建军, 男, 1979 年生, 博士, 助理研究员; E-mail: wang0997105@hotmail.com.

** 通讯作者; E-mail: jishen@niglas.ac.cn. 
但如其他所有方法一样，以上两大类方法存在各自的局限性. 虽然原位箱式法及沉积物柱样培养法 均可采用一定的扰动方式来代替水动力扰动, 但是, 该类模拟的扰动方式形成的水流等, 很难与实际情 形下类似, 从而测得的沉积物耗氧速率有可能不能代表原位情况下的实际数值 ${ }^{[4]}$. 而底层扩散边界层氧 气含量剖面只能获得一维或者二维的氧气图像，对生物扰动作用 (bioturbation) 和生物灌溉作用 (bioirrigation)可能会有所低估.

近年来，也有新方法出现，如涡度-相关性法(eddy-correlation method $)^{[12-13]}$. 该方法不破坏表层沉积物， 且与氧气在沉积物-水界面的迁移机制无关; 但该方法具有类似第一种方法的缺陷, 其通过测定固定面积 条件下氧气变化, 仅能获得 TOE. 此外, 除该类方法之外, 也可以通过获得体积式氧气交换速率来间接获得 沉积物氧气交换速率, 该方法被 de Beer 等引进 ${ }^{[7]}$, 并与其他方法进行比较验证 ${ }^{[14]}$, 主要应用于渗透性的沉 积物(如沙质性海洋沉积物)中. 从另一个角度, 我们推测, 在湖泊沉积物及扩散边界层(Diffusive Boundary Layer, DBL)的特定层位中, 同样也应该存在着特定的体积式氧气交换速率: 即随着上覆水水流的停止, 沉 积物及 DBL 层某一层位的氧气含量在水流停止后的短时间内呈线性下降，且其斜率是固定的.

$\mathrm{OE}$ 容易受到很多因素影响. 比如温度能够通过改变氧气在水体中的溶解度, 改变分子运动势能及改 变生物活性来影响 $\mathrm{OE}^{[15-18]}$. 此外, 底栖微藻的光合作用会造成 $\mathrm{OE}$ 白天和晚上的差异 ${ }^{[19]}$. 底栖生物本身 的呼吸作用, 灌溉作用等, 会增加 $\mathrm{OE}^{[2,20-23]}$. 而在空间尺度上，由于沉积物本身的物理化学以及生物性质 的不同，如沉积物有机质含量 ${ }^{[24]}$, 从而形成 $\mathrm{OE}$ 在空间上的异质性. 研究 $\mathrm{OE}$ 异质性的同时, 借以不同的 方法来考察 $\mathrm{OE}$, 则可以获得一些生物化学因素对界面 $\mathrm{OE}$ 的影响程度. 比如, 底栖生物的呼吸速率, 可通过 其生物量和实验室内测定的单位生物量耗氧速率来计算 ${ }^{[25]}$; 底栖生物带来的交换速率影响, 可由 TOE 与 $\mathrm{DOE}$ 的差额计算获得 ${ }^{[26]}$. 因此, 在实验过程中，根据实验目的，采用不同的方法测定界面 $\mathrm{OE}$ 具很大的必 要性.

本研究根据水流开启-停止过程中 DBL 层氧气随时间变化的规律，检验该方法在 OE 测定中的应用; 此外, 选择不同湖泊的十个位点, 采集获得在沉积物物理化学性质具有较大差异沉积物柱状样品, 在实 验室内通过溶氧微电极法和沉积物柱样培养法测定 $\mathrm{OE}$, 并考察影响 $\mathrm{OE}$ 在空间尺度上差异性的因素.

\section{1 材料与方法}

沉积物柱样采用柱样采集器(自制, $\Phi=90 \mathrm{~mm}$ )获得, 分别来自于山东南四湖及江苏太湖, 具体采样时 间及位点见表 1 ; 每个位点获柱样 3 个, 同时采集 $20 \mathrm{~L}$ 上覆水用于室内培养. 样品采集后, 尽量少扰动地 运回南京, 并避光放置在自制沉积物柱样孵育系统中(专利号 ZL200720043927.9), 调节水温至采样时的

表 1 采样位点概况

Tab.1 Sampling sites

\begin{tabular}{llllll}
\hline 采样点 & 北纬 & 东经 & 时间 & 水深(m) & 其他 \\
\hline M1 & $31^{\circ} 32^{\prime} 02.4^{\prime \prime}$ & $120^{\circ} 12^{\prime} 48.1^{\prime \prime}$ & $2005-10-01$ & 2.1 & 太湖梅梁湾北部三山岛 \\
M2 & $31^{\circ} 29^{\prime} 03.0^{\prime \prime}$ & $120^{\circ} 11^{\prime} 56.0^{\prime \prime}$ & $2005-10-01$ & 2.5 & 太湖梅梁湾湖心 \\
M3 & $31^{\circ} 23^{\prime} 58.1^{\prime \prime}$ & $120^{\circ} 11^{\prime} 31.3^{\prime \prime}$ & $2005-10-01$ & 2.0 & 太湖梅梁湾湾口 \\
M4 & $31^{\circ} 17^{\prime} 38.2^{\prime \prime}$ & $120^{\circ} 11^{\prime} 4.9^{\prime \prime}$ & $2005-10-01$ & 2.7 & 太湖湖心 \\
S1 & $35^{\circ} 00^{\prime} 50.2^{\prime \prime}$ & $116^{\circ} 50^{\prime} 54.2^{\prime \prime}$ & $2005-09-28$ & 2.4 & 南四湖(独山湖区) \\
S2 & $34^{\circ} 58^{\prime} 17.8^{\prime \prime}$ & $116^{\circ} 52^{\prime} 53.4^{\prime \prime}$ & $2005-09-28$ & 3.2 & 南四湖(独山湖区) \\
S3 & $34^{\circ} 37^{\prime} 55.0^{\prime \prime}$ & $117^{\circ} 15^{\prime} 33.0^{\prime \prime}$ & $2005-09-29$ & 3.2 & 南四湖(微山湖区) \\
S4 & $34^{\circ} 45^{\prime} 11.7^{\prime \prime}$ & $117^{\circ} 04^{\prime} 42.1^{\prime \prime}$ & $2005-09-29$ & 4.0 & 南四湖(微山湖区) \\
S5 & $34^{\circ} 45^{\prime} 04.5^{\prime \prime}$ & $117^{\circ} 05^{\prime} 43.9^{\prime \prime}$ & $2005-09-29$ & 2.0 & 南四湖(微山湖区) \\
S6 & $34^{\circ} 12^{\prime} 22.9^{\prime \prime}$ & $117^{\circ} 37^{\prime} 11.3^{\prime \prime}$ & $2005-09-27$ & 3.0 & 南四湖, 洙赵新河人湖口 \\
\hline
\end{tabular}


湖水平均温度 $20^{\circ} \mathrm{C}$; 磁转子位于沉积物表层上方 $8-10 \mathrm{~cm}$ 处, 扰动强度约为 80 转 $/$ 分. 在培养过程中, 间 断性曝气(经饱和 $\mathrm{NaOH}$ 溶液过滤的空气)，使上覆水水体含氧量在实验期间保持相对恒定.

界面氧气垂直分布的测定采用溶氧微电极进行(PreSens, 德国). 电极的有效测定区(sensing tip)小于 $20 \mu \mathrm{m}$, 涂有固态光敏荧光粉; 在 $0 \%-100 \%$ 空气饱和度水体中, 氧气浓度与光强呈线性关系; 电极反应时 间小于 $1 \mathrm{~s}$; 每次使用前, 采用饱和空气 $(100 \%$ 氧气 $)$ 与加人 $\mathrm{Na}_{2} \mathrm{SO}_{3}$ 的纯净水 $(0 \%$ 氧气 进行两点校准. 电极 安装在微电极操作器(专利号 ZL200720043928.3)上, 每 $5 \mathrm{~s}$ 下移电极 $0.02 \mathrm{~mm}$ 进行连续测定, 直至氧气含 量接近于 $0.0 \mathrm{mg} / \mathrm{L}$ 为止. 为准确定位微电极在沉积物-水的位置, 采用放大镜进行裸眼观察.

氧气在 DBL 层中的交换速率(DOE $)^{[2,2]}$ 采用 Fick 定律来计算, 计算公式如下:

$$
D O E=D_{0}(\mathrm{~d} C(z) / \mathrm{d} z)
$$

式中, $D_{0}$ 为一定温度和盐度下氧气在水体中的扩散系数, $C(z)$ 为 DBL 的某一个深度 $z$ 条件下氧气的含量. DOE 负值表示沉积物氧气的净消耗, 而正值表示氧气从沉积物释放进人上覆水水体.

此外, 通过对沉积物中氧气剖面进行模型拟合, 得氧气在沉积物中的扩散速率 $\left(\mathrm{DOE}_{\mathrm{s}}\right)^{[2]}$, 计算公式 如下:

$$
D O E_{\mathrm{s}}=D_{\mathrm{s}}(\mathrm{d} C(z) / \mathrm{d} z)_{\text {Sedi }}
$$

式中, $D_{\mathrm{s}}$ 为在沉积物中氧气在一定温度下的有效扩散系数, $C$ 为沉积物的某一深度 $z$ 氧气的含量. $D O E_{\mathrm{s}}$ 负 值表示沉积物氧气的净消耗, 而正值表示氧气从沉积物释放进人上覆水. 由于本实验均是在无光照的条 件下进行的, 因此, 所有的 $D O E$ 和 $D O E_{\mathrm{s}}$ 均为负值, 即表现为沉积物对氧气的消耗, 因此, 为简便起见, 在本文中谈及的 $D O E, D O E_{\mathrm{s}}$ 等界面氧气交换速率均取其绝对值，而省去负号.

DBL 中体积式氧气交换速率 $\left(\mathrm{O}_{2}(t)\right.$ ) 首先在沙质性海洋沉积物中使用 ${ }^{[7]}$, 其基本原理是, 让具有一定浓 度的氧气以恒流流经沙质性海洋沉积物, 然后突然停止水流, 连续测定沉积物不同深度氧气浓度随时间变 化的速率; 在水流停止后较短时间内 (如 $10 \mathrm{~min}$ ), 氧气含量的变化速率是恒定的. 而在湖泊沉积物中, 这种 方法却难以应用, 因为类似的沙质性沉积物较为少见. 因此, 本实验是直接测定 DBL 层, 而非沉积物间 隙水中的氧气含量. 由于 DBL 层的一般厚度在 0.0-1.0 $\mathrm{mm}$ 之间 ${ }^{[27]}$, 所以将微电极移动至沉积物表层上方 $0.2 \mathrm{~mm}$ 处持续记录在扰动停止后氧气含量. 该处起始氧气含量随时间的线性拟合斜率则为 $\mathrm{O}_{2}(t)$ (单位: $\mu \mathrm{mol} /(\mathrm{L} \cdot \mathrm{min}))$.

界面氧气交换速率测定前, 每个柱状样至少平衡超过 $12 \mathrm{~h}$, 并以关闭柱样上盖为起始. 根据培养系统 中的水温, 整个培养时间在 1-12h 之内结束, 在培养期间内, 采用溶解氧电极每 $5 \mathrm{~min}$ 测定上覆水水体的 溶解氧含量. 一般而言, 需保证测定过程中上覆水水体的氧气含量不低于初始氧气含量的 $20 \%^{[28-29]}$, 因 为这段时间内, 上覆水水体中的氧气含量基本保持线性下降. 电极在测定前进行校准, 随后采用同样的 氧电极测定所有的柱样. 在实验结束后, 计算 TOE, 公式如下:

$$
T O E=\left(\alpha \times V_{1}\right) / A
$$

其中, $\alpha$ 是单位时间内上覆水氧气含量的变化量, $V_{1}$ 为上覆水体积, $A$ 为沉积物表面积.

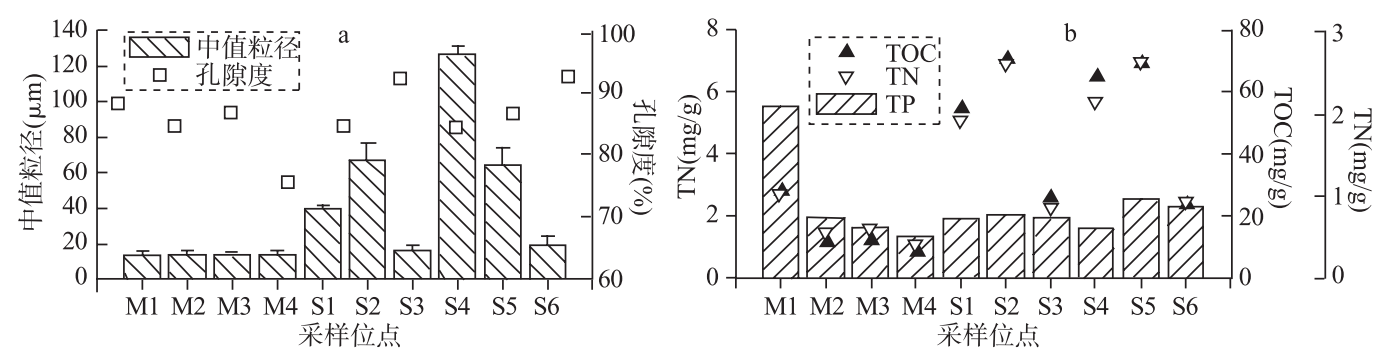

图 1 采样位点沉积物理化学性质

Fig.1 Physical and chemical characters of sampled sediments 
沉积物总有机碳 (TOC), 总氮 (TN) 和总磷(TP)的测定分别采用 $\mathrm{K}_{2} \mathrm{CrO}_{7}-\mathrm{H}_{2} \mathrm{SO}_{4}$ (油浴)氧化- $\mathrm{FeSO}_{4}$ 滴定, $\mathrm{K}_{2} \mathrm{CrO}_{7}-\mathrm{H}_{2} \mathrm{SO}_{4}$ 消化-凯氏定氮法和 $\mathrm{HClO}_{4}-\mathrm{H}_{2} \mathrm{SO}_{4}$ 酸溶-钼锑抗比色法. 沉积物粒度测定采用 Malven Mastersizer 2000 , 计算获得中值粒径. 孔隙率 $(\Phi, \mathrm{V} / \mathrm{V})$ 来自于 $105^{\circ} \mathrm{C}$ 烘 $4 \mathrm{~h}$ 前后损失水的体积与烘干前沉积 物体积的比值. 沉积物扩散系数 $D_{\mathrm{s}}$ 由沉积物孔隙率计算获得:

$$
D_{\mathrm{s}}=D_{0} /(1+3(1-\Phi))
$$

\section{2 结果}

\section{1 沉积物性质}

2.1.1 物理性质 太湖沉积物中值粒径显著小于南四湖(学生 $t$-检验, $P<0.05$ ) (图 1a). 如太湖的中值粒径平 均值为 $13.31 \mu \mathrm{m}$, 而南四湖的则为 $55.24 \mu \mathrm{m}$; 太湖四个位点的中值粒径之间不具有显著性差异 $(P>0.05)$, 最小值为 $12.80 \mu \mathrm{m}(\mathrm{M} 1)$, 最大值为 $13.74 \mu \mathrm{m}(\mathrm{M} 4)$. 南四湖湖泊沉积物中值粒径变化较大, 最小值为 $15.66 \mu \mathrm{m}(\mathrm{S} 3)$, 最大值为 $126.31 \mu \mathrm{m}(\mathrm{S} 4)$. 两个湖泊的沉积物孔隙度不具有显著性差异 $(P<0.05)$ (图 1a). 太 湖沉积物孔隙度从梅梁湾北部的河口至太湖湖心, 呈下降趋势; 以河口最大, 为 $88.28 \%$, 而湖心较低, 仅为 $75.58 \%$. 南四湖沉积物孔隙度不具有明显规律，最大值为 $92.63 \%$, 最小值为 $84.42 \%$.

2.1 .2 化学性质 沉积物总有机碳(TOC)与总氮 (TN)之间具显著相关性 $(P<0.001$, 线性拟合). 太湖 $\mathrm{TOC}$, TN 以及 TP 从梅梁湾北部的河口至太湖湖心，均呈明显的下降趋势(图 1b), 河口沉积物含量最高，而湖心 最低. 南四湖沉积物 TOC, TN 和 TP 之间的变化幅度较大. 如 TOC 最大值为 $70.65 \mathrm{mg} / \mathrm{g}(\mathrm{S} 2)$, 最小值为 $23.74 \mathrm{mg} / \mathrm{g}(\mathrm{S} 6)$. 与太湖相比, 南四湖沉积物 TOC 和 TN 含量显著大于太湖 $(P<0.05)$; 南四湖 TP 最大值为 $0.96 \mathrm{mg} / \mathrm{g}(\mathrm{S} 5)$, 最小值为 $0.60 \mathrm{mg} / \mathrm{g}(\mathrm{S} 4)$. 需要提及的是, 南四湖 TP含量均远小于太湖梅梁湾河口 M1 的数 值 $(2.09 \mathrm{mg} / \mathrm{g})$; 而整体而言南四湖沉积物 TP 与太湖差异不显著 $(P>0.05)$.

\section{2 氧气微剖面测定界面氧气交换速率}

太湖和南四湖 10 个位点的氧气微剖面均表现为由上覆水向下，随着深度增加溶解氧浓度逐渐降低; 这 现象与其他相关研究结果类似 ${ }^{[4]}$, 表明湖泊沉积物一直保持着消耗氧气的状态. 氧气侵蚀深度 (Oxygen Penetration Depth, OPD) 均值为 $5.34 \mathrm{~mm}$, 变化范围在 3.92-7.60mm 之间(图 2), 且两个湖泊之间不存在显 著差异 $(P>0.05$, 学生 $t$-检验).

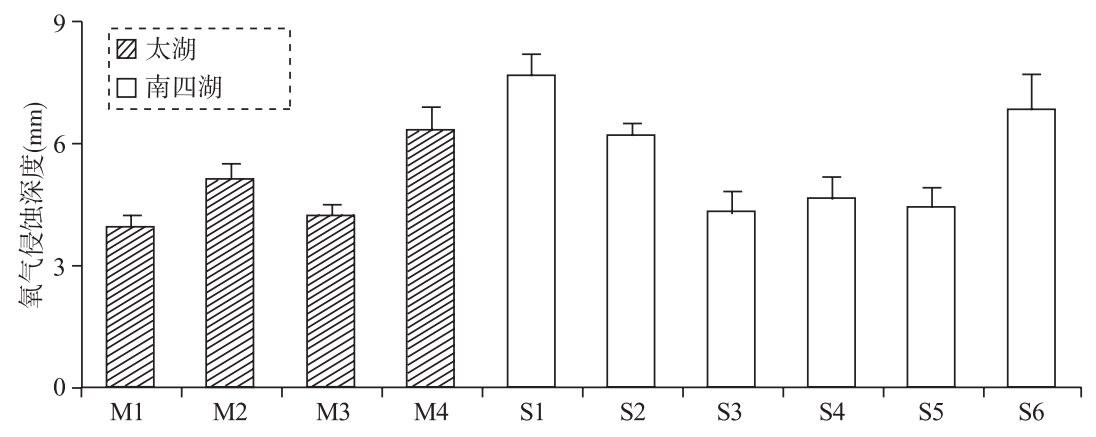

图 2 采样位点氧气侵蚀深度

Fig.2 Oxygen penetration depth at each site

根据 DBL 层的氧气剖面斜率 ${ }^{[2]}$, 计算出 DOE(图 3d). 太湖 4 个位点中, DOE 在梅梁湾北部的位点 $\mathrm{M} 1$ 最高, 为 $764.94 \mu \mathrm{mol} /\left(\mathrm{m}^{2} \cdot \mathrm{h}\right)$, 梅梁湾湾口 $\mathrm{M} 3$ 最低, 为 $291.45 \mu \mathrm{mol} /\left(\mathrm{m}^{2} \cdot \mathrm{h}\right)$. 南四湖 DOE 平均值为 $554.07 \mu \mathrm{mol} /\left(\mathrm{m}^{2} \cdot \mathrm{h}\right)$, 变化范围为 $263.00-1021.31 \mu \mathrm{mol} /\left(\mathrm{m}^{2} \cdot \mathrm{h}\right) .2$ 个湖泊的 DOE 数值之间无显著差异 $(P>0.05)$. 对沉积物中氧气剖面的零阶拟合 ${ }^{[2]}$, 计算出 $\mathrm{DOE}_{\mathrm{s}}$ (图 3c). 太湖 4 个位点 $\mathrm{DOE}_{\mathrm{s}}$ 自北部 $\mathrm{M} 1$ 至南 部 M4 逐渐降低. 变化范围为 $1393.90-396.17 \mu \mathrm{mol} /\left(\mathrm{m}^{2} \cdot h\right)$. 南四湖 DOE 以 S1 最高, 为 $1123.44 \mu \mathrm{mol} /\left(\mathrm{m}^{2} \cdot \mathrm{h}\right)$, $\mathrm{S} 6$ 其次, 为 $895.32 \mu \mathrm{mol} /\left(\mathrm{m}^{2} \cdot \mathrm{h}\right), \mathrm{S} 4$ 最低, 为 $447.12 \mu \mathrm{mol} /\left(\mathrm{m}^{2} \cdot \mathrm{h}\right)$. 与 $\mathrm{DOE}$ 一样, 2 个湖泊的 $\mathrm{DOE}_{\mathrm{s}}$ 数值之间 
也无显著差异 $\left(P>0.05\right.$, student $t$-test). $\mathrm{DOE}_{\mathrm{s}}$ 与 $\mathrm{DOE}$ 相比较, 前者数值大部分高于或接近后者. 比如, $\mathrm{S} 6$ 的 $\mathrm{DOE}$ 仅是 $\mathrm{DOE}_{\mathrm{s}}$ 的 $30.3 \%, \mathrm{M} 1$ 为 $54.9 \%$, 而 S4 位点为 $99.1 \%$. 但是, 有 2 个位点(S2 和 S5)DOE 较 $\mathrm{DOE}_{\mathrm{s}}$ 高, 高出的份额分别为 $7.1 \%$ 和 $22.0 \%$.

\section{3 沉积物柱样培养法测定界面氧气交换速率}

采用沉积物柱样培养法, 根据培养过程中上覆水氧气含量的斜率, 计算出 TOE(图 3b). 在所有的沉 积物柱状样品中, 密闭环境条件下，上覆水氧气含量在初始时间内，均随着时间呈线性下降，这表明沉 积物对氧气的消耗, 且该消耗是可以进行定量的. 太湖的 4 个位点, 自北部的 M1 点至南部的 M4 点, TOE 逐渐下降, 其变化范围为 $2183.77-484.25 \mu \mathrm{mol} /\left(\mathrm{m}^{2} \cdot \mathrm{h}\right)$. 南四湖 TOE 变化范围为 $588.23-2102.81 \mu \mathrm{mol} /\left(\mathrm{m}^{2} \cdot \mathrm{h}\right)$. 最高值发生在 S2 位点, 而最低值发生在 S3 位点. 南四湖的 TOE 均值较太湖高, 分别为 $1365.40 \mu \mathrm{mol} /\left(\mathrm{m}^{2} \cdot \mathrm{h}\right)$ 和 $1098.81 \mu \mathrm{mol} /\left(\mathrm{m}^{2} \cdot \mathrm{h}\right)$, 但是两湖之间不存在显著差异 $(P>0.05$, 学生 $t$-检验 $)$.
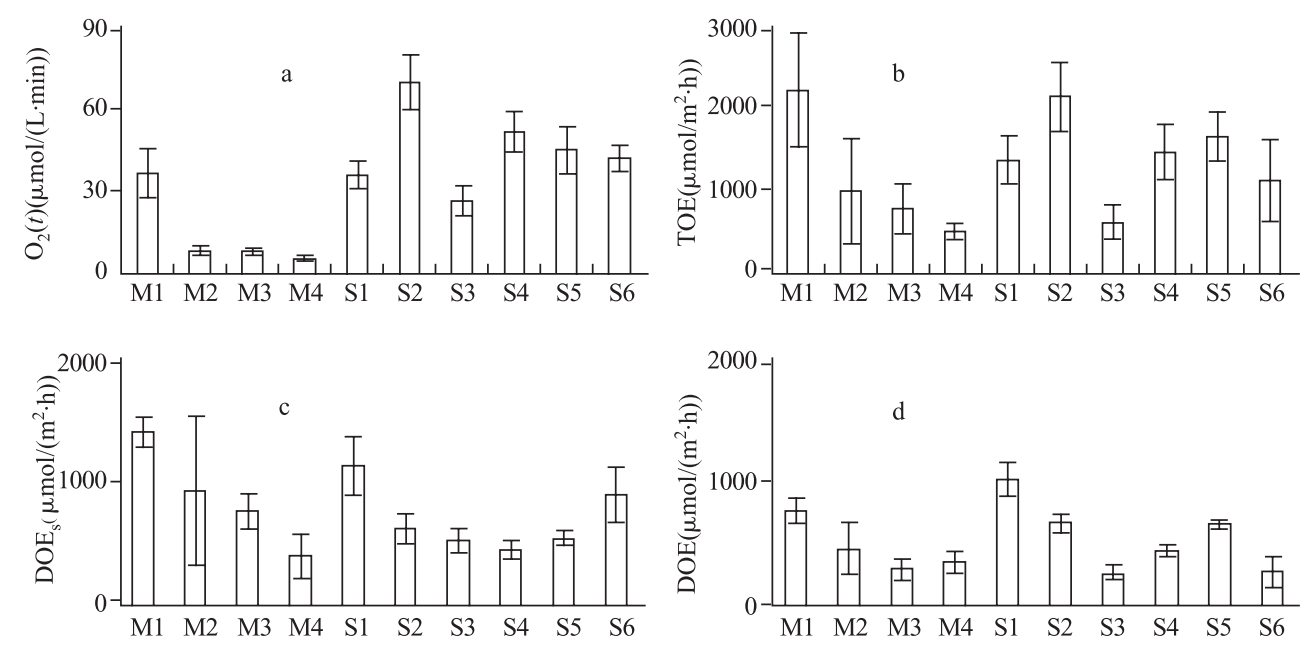

图 3 各位点的沉积物-水界面氧气交换 $\left(\mathrm{a}: \mathrm{O}_{2}(t) ; \mathrm{b}: \mathrm{TOE} ; \mathrm{c}: \mathrm{DOE}_{\mathrm{s}}\right.$; d: DOE, 每个图含标准误差线)

Fig.3 Oxygen exchange at the sediment-water interface with error bars(a: $\mathrm{O}_{2}(t)$; b: TOE; c: DOE $;$; d: DOE)

\section{4 水流启动-停止法测定界面氧气交换速率}

采用水流启动-停止法, 可获水流停止后 DBL 层的某一个位置氧气随时间变化的曲线; 根据曲线的 斜率, 可进一步获得该位点的体积式界面氧气交换速率 $\mathrm{O}_{2}(t)$. 采用此方法对太湖及南四湖所有的样品在 原位水温条件下进行 $\mathrm{O}_{2}(t)$ 测定, 结果如图 3a 所示. 太湖梅梁湾湖心 $\mathrm{M} 2$ 至南部的 $\mathrm{M} 4, \mathrm{O}_{2}(t)$ 逐渐降低, 其

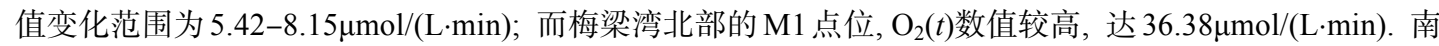
四湖 $\mathrm{O}_{2}(t)$ 变化范围为 $26.45-69.34 \mu \mathrm{mol} /(\mathrm{L} \cdot \mathrm{min})$, 其均值为 $44.99 \mu \mathrm{mol} /(\mathrm{L} \cdot \mathrm{min})$, 比太湖的 $\mathrm{O}_{2}(t)$ 均值高很多 (太湖 $14.45 \mu \mathrm{mol} /(\mathrm{L} \cdot \mathrm{min})$ ); 统计结果表明, 太湖 $\mathrm{O}_{2}(t)$ 与南四湖之间差异性显著 $(P=0.012$, 学生 $t$-检验).

\section{3 讨论}

\section{1 几种方法获得的界面氧气交换速率之间的差异}

通过模型拟合溶解氧微剖面常常用来获得界面氧气交换速率，如 Rasmussen 和 Jørgensen ${ }^{[2]}$ 通过拟合 溶解氧微剖面的 DBL 层和沉积物中的氧气含量计算获得 DOE 和 $\mathrm{DOE}_{\mathrm{s}}$; 他们的结果表明, 这两种计算方 法可获得较为类似的结果. 在本研究中, 采用这两种方法计算出 $\mathrm{DOE}$ 和 $\mathrm{DOE}_{\mathrm{s}}$, 同样发现 $\mathrm{DOE}_{\mathrm{s}}$ 的数值较 DOE 大，且两者之间差异不显著 $(P>0.05$, 学生 $t$-检验).

DBL 层较沉积物本身而言, 有其内在的缺点, 如易受到水流和扰动因素影响 ${ }^{[6]}$. 但 DBL 层不会受到沉积 物中的孔隙度和曲折度的影响. 沉积物中存在垂向孔隙度差异, 从而有可能造成 $\mathrm{DOE}_{\mathrm{s}}$ 计算结果的误差 ${ }^{[5,30]}$. 此外, 通过沉积物中的氧气微剖面进行 $\mathrm{DOE}_{\mathrm{s}}$ 计算时, 零阶拟合过于简单, 从而有可能过高估算 $\mathrm{DOE}_{\mathrm{s}}$ 的值. 
沉积物中存在还原性产物 ${ }^{[31]}, \mathrm{NH}_{4}{ }^{+}, \mathrm{Fe}^{2+}$ 等扩散进人沉积物氧化层, 进一步被氧气氧化, 从而改变氧气的微 剖面状态. 有资料表明, 表层沉积物中的易降解性有机物质, 能够使得 DBL 以下的斜率增加, 从而容易得 到偏高的氧气交换速率 ${ }^{[6,32]}$. DBL 层氧气剖面的影响因素较为简单, 而沉积物中的影响因素较为复杂, 且在 进行 $\mathrm{DOE}_{\mathrm{s}}$ 计算时的线性模型过于简单, 这些因素可能是造成 $\mathrm{DOE}_{\mathrm{s}}$ 数值较 $\mathrm{DOE}$ 高的原因.

但是，上述论述并不表示 DOE 的数值能够真正代表整个沉积物的氧气交换速率，这是因为, DOE 提 供的信息仅包括由分子扩散速率而得出的部分, 并不包括生物扰动, 生物呼吸以及生物灌溉等作用而提 升的氧气交换速率 ${ }^{[3]}$. 而且, 在采用微电极法进行界面氧气交换速率计算时, 常采用的是一维氧气分布 数据, 而很少会涉及到二维或三维分布数据. 实际上, 沉积物的三维地形结构会提高沉积物氧气交换速 率 ${ }^{[4,33]}$. 研究结果表明, TOE 数值与 DOE 或者 DOEs 差异显著 $(P<0.05$, 学生 $t$-test), 而且后者仅是前者的 $41.2 \%-60.6 \%$. 这与其他很多结果类似. Andersson 和 Helder ${ }^{[34]}$ 在潮间带沉积物的研究发现, TOE 是 DOE 的 1.4 至 3.2 倍. Rasmussen 和 Jørgensen ${ }^{[2]}$ 在 Aarhus 湾等沉积物的研究发现, DOE 平均占 TOE 的 $45 \%$, 并 表明 TOE/DOE 比值在 1.5-2.6 范围内变化. Kim 等 ${ }^{[24]}$ 在 Taean 湾的结果也表明 TOE/DOE 比值为 2-3. 但 是, 也有研究表明 TOE 与 DOE 之间数值较为相近 ${ }^{[35]}$. 本研究中的湖泊水体较浅, 而且沉积物中富含底栖 动物. 因此除扩散作用之外, 界面氧气交换速率还将受到其他多种因素影响, 从而导致 TOE 数值高于 DOE 数值的现象. 由此可见, 通过沉积物柱样培养法或原位箱式法获得 TOE 数值才能更接近真实的体现 湖泊沉积物的氧气交换速率; DOE 仅仅只能用来代表由分子扩散而形成的氧气交换速率; 而 TOE 与 DOE 的差值,可表征由底栖动物带来的氧气交换速率的信息 ${ }^{[26]}$.

本研究采用水流启动-停止法来测定沉积物界面氧气交换速率 $\left(\mathrm{O}_{2}(t)\right)$. 在应用这一方法之前, 该方法 的可靠性需验证. 流经柱样沉积物的水流速度为 $1.0 \mathrm{~cm} / \mathrm{s}$ 左右, $4.8^{\circ} \mathrm{C}$ 条件下, 能获得 $>0.40 \mathrm{~mm}$ 的 $\mathrm{DBL}$ 层; $25.2^{\circ} \mathrm{C}$ 条件下, 能获得 $>0.2 \mathrm{~mm}$ 的 DBL 层. 每次进行水流终止前, 该水流至少保持 5-10 min, 使得氧气侵 蚀深度和 DBL 层深度达到稳定. 然后, 突然中断水流. 在此过程中, 持续记录水流开启状态时和水流停 止后 DBL 层某一深度(如 $0.2 \mathrm{~mm}$ )氧气浓度的变化情况. 然后重复上述操作多次. 结果表明, 该方法针对 某一样品而言, $\mathrm{O}_{2}(t)$ 具有很高的重复性(数据未给出), 这表明该数值能够特征性的表征该样品的氧气消耗 速率. 但是, 在此需要提及的是, 对于同一个柱状样品而言, 不同的 DBL 层深度, $\mathrm{O}_{2}(t)$ 的数值不同; 因此, 在实际应用过程中, $\mathrm{O}_{2}(t)$ 数值必须与特定的 DBL 位置结合起来, 本研究选择 DBL 层中的 $0.2 \mathrm{~mm}$ (即沉积 物表层上方 $0.2 \mathrm{~mm}$ ) 来进行样品 $\mathrm{O}_{2}(t)$ 间的比较研究.

$\mathrm{O}_{2}(t)$ 与其他界面氧气交换速率的单位不同. $\mathrm{O}_{2}(t)$ 代表一种体积式的氧气交换速率，表示为在一定时 间内, 某一位点的氧气浓度变化速率. 假如将 $\mathrm{O}_{2}(t)$ 应用在沉积物中, 那就表示在沉积物氧化层的某一位 点, 氧气的消耗速率. 而将 $\mathrm{O}_{2}(t)$ 应用于 DBL 层中, DBL 中的 $\mathrm{O}_{2}(t)$ 则可表征沉积物的呼吸代谢特征. 由于 DBL 层不受沉积物本身物理性质的影响, 因此 DBL 层中的氧气交换主要是由 DBL 层上方上覆水水体中 的氧气补给和下方沉积物中氧气消耗两种机制联合作用而形成的一种动态平衡. 实验表明, 在水流停止 后的短时间内, DBL 层中的氧气消耗呈线性下降，且该拟合曲线具有固定斜率. 因此，根据该斜率与 DBL 层氧气消耗保持一致且具较高重复性的原理, 可使用 $\mathrm{O}_{2}(t)$ 来表征沉积物的氧气交换速率. 与氧气微剖面 获得的结果不同的是, $\mathrm{O}_{2}(t)$ 不仅包含扩散作用，而且也包括生物扰动在内的很多其他因素; 该点可由 $\mathrm{O}_{2}(t)$ 与 $\mathrm{TOE}$ 的显著相关性获得直接证实(图 4). 因此, 较 $\mathrm{DOE}$ 和 $\mathrm{DOE}_{\mathrm{s}}$ 而言, $\mathrm{O}_{2}(t)$ 能够更加真实地表征沉积物 呼吸特征, 且其数值能够与传统柱样培养法获得的 TOE 较好吻合.

\section{2 界面氧气交换速率的空间异质性及其因素分析}

由于沉积物本身存在异质性, 如有机物质的局部聚集(Organic Hot Spots) ${ }^{[36]}$, 而与之相对应的是, 采 用微电极技术, 只能获取很小范围内的氧气速率. 这种矛盾, 有可能引起氧气微剖面获得数据不能真实 反映实际的界面氧气交换速率. 此外, 由微地形结构引起的 DBL 层厚度的差异也可形成较小范围内界面 交互速率的差异 ${ }^{[6,27]}$. 如在 $2 \mathrm{~mm}$ 的沉积物范围内, 氧气的生成和释放可能分别出现 3 和 20 倍的差异 ${ }^{[37]}$. 但是, 也有研究表明, 在不考虑光照的条件下, 氧气微剖面可获得真实的界面扩散速率 ${ }^{[3,18]}$. 本研究中, 由每个位点 3-7 个氧气微剖面获得的 DOE 和 $\mathrm{DOE}_{\mathrm{s}}$ 均具有较小的标准误差(图 3c, 图 3d), 这表明微氧电 极获取的界面扩散速率信息具较大的可靠性. 


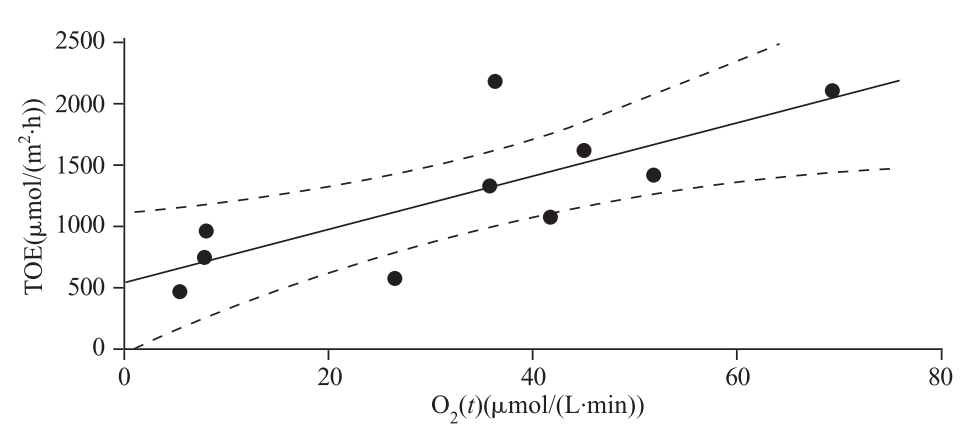

图 $4 \mathrm{TOE}$ 与 $\mathrm{O}_{2}(t)$ 之间的关系(实线为线性拟合, $P<0.01$; 虚线为线性拟合 $95 \%$ 置信区间)

Fig.4 The relationship between TOE and $\mathrm{O}_{2}(t)$

(Solid line: linear regression, $P<0.05$; Dotted line: $95 \%$ confidence intervals)

界面物质交换速率与氧气侵蚀深度密切关联. 但本研究中 $\mathrm{DOE}_{\mathrm{s}}$ 与 $\mathrm{OPD}$ 之间并不存在显著的负相关 性, 即, 随着 OPD 的降低, $\mathrm{DOE}_{\mathrm{s}}$ 增加. 同样, $\mathrm{DOE}$ 与 $\mathrm{OPD}$ 也不具有显著的负相关性 $(P>0.05)$. 这种现象, 可能与沉积物表层有机物质性质等不明原因有关. 比如, 在好氧性沉积物中, 当沉积物表层有机质较容 易被好氧分解的情况下, 氧气在该层降低的斜率较大, 导致 $\mathrm{DOE}_{\mathrm{s}}$ 和 $\mathrm{DOE}$ 均较大; 而在该层以下的好氧 性沉积物中, 由于有机质不容易好氧分解, 则表现为氧气斜率逐渐变缓; 该层斜率变缓的快慢, 则会表 现为 $\mathrm{OPD}$ 数值的差异 ${ }^{[32]}$.

室内试验过程中，上覆水氧气含量基本保持一致，而且各样品之间扰动或者水流速度也保持一致; 因此, 初始氧气含量和水流速度并不会造成对目前实验结果的影响, 而影响异质性的因素仅来自于沉积 物本身的物理化学以及生物性质, 如有机物质的可降解性, 底栖动物的数量和群落结构以及还原性化学 物质的含量等因素的影响 ${ }^{[36]}$. 本结果中有趣的是, TOE 和 $\mathrm{O}_{2}(t)$ 确实与沉积物 TOC/TN 之间具有显著的相 关性 $(P<0.01)$, 而 $\mathrm{DOE} / \mathrm{DOE}_{\mathrm{s}}$ 与 $\mathrm{TOC} / \mathrm{TN}$ 之间的相关性则不明显(图 5). 其他参数, 如粒度、 $\mathrm{TP}$ 、孔隙度 等, 与上述界面氧气交换速率参数相关性不显著. 可惜的是, 本研究并没考察沉积物中底栖动物的数量 和生物量. 根据 2007 年对太湖同一位点底栖动物群落的研究(未发表数据), 结果表明太湖北部梅梁湾 M1 至

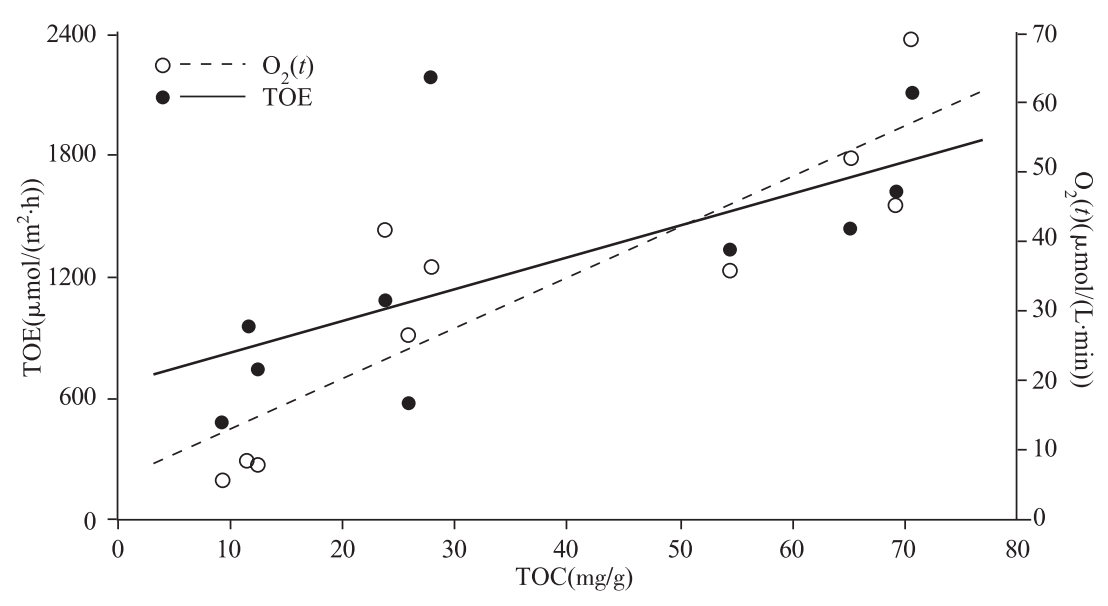

图 5 沉积物-水界面氧气交换速率与沉积物总有机碳之间的关系 (其中, TOE-TOC, 线性拟合, $P<0.05 ; \mathrm{O}_{2}(t)-\mathrm{TOC}$, 线性拟合, $P<0.01$; $\mathrm{DOE}, \mathrm{DOE}_{\mathrm{s}}$ 与 $\mathrm{TOC}$ 之间的关系不显著, 图未给出)

Fig.5 The linear relationship between oxygen exchange and sediment TOC (Solid line: $P<0.05$; Dotted line: $P<0.01$ ) 
太湖湖心 M4, 底栖生物以水蚯蚓为主, 且底栖动物数量及生物量逐渐降低. 根据现有结果, 可以认为, 本研究涉及的各位点在界面氧气交换速率的差异主要受到沉积物有机物质(如 TOC, TN)含量的影响.

\section{4 结论}

水流启动-停止的方法，可有效获得体积式沉积物耗氧速率 $\mathrm{O}_{2}(t)$, 且该速率与 TOE 之间具有较高相 关性; 与 $\mathrm{DOE}$ 和 $\mathrm{DOE}_{\mathrm{s}}$ 数值相比, $\mathrm{O}_{2}(t)$ 数值不仅能代表氧气扩散速率, 且包括沉积物中生物呼吸以及生物 扰动引起的氧气交换速率信息. 值得注意的是，界面氧气交换速率测定方法和实验条件对测定结果影响 较大，因此在进行界面氧气交换速率测定的过程中，不仅测定手段需仔细选择，而且在整个实验设计中， 实验条件也需保持恒定，从而保证实验过程中结果的一致性和可比性. 通过多位点不同沉积物性质条件 下界面氧气交换速率的研究，结果表明界面氧气交换速率的空间差异性，除表现在生物因素，如生物扰 动，生物灌溉和生物本身的呼吸作用外，还表现在沉积物有机质含量上，如有机碳含量等.

\section{5 参考文献}

[1] Nixon SW. Coastal marine eutrophication: A definition, social causes, and future concerns. Ophelia, 1995, 41: 199-219.

[2] Rasmussen H, Jørgensen BB. Microelectrode studies of seasonal oxygen uptake in a coastal sediment: Role of molecular diffusion. Marine Ecology Progress Series, 1992, 81(3): 289-303.

[3] Wenzhöer F, Glud RN. Small-scale spatial and temporal variability in coastal benthic $\mathrm{O}_{2}$ dynamics: Effects of fauna activity. Limnology and Oceanography, 2004, 49(5): 1471-1481.

[4] Jørgensen BB, Marais DJD. The diffusive boundary layer of sediments: oxygen microgradients over a microbial mat. Limnology and Oceanography, 1990, 35(6): 1343-1355.

[5] Lansard B, Rabouille C, Massias D. Variability in benthic oxygen fluxes during the winter-spring transition in coastal sediments: an estimation by in situ micro-electrodes and laboratory mini-electrodes. Oceanologica Acta, 2003, 26(3): $269-279$.

[6] Gundersen JK, Jørgensen BB. Microstructure of diffusive boundary layers and the oxygen uptake of the sea floor. Nature, 1990, 345(6276): 604-607.

[7] de Beer D, Wenzhofer F, Ferdelman TG et al. Transport and mineralization rates in North Sea sandy intertidal sediments, Sylt-Rømø Basin, Wadden Sea. Limnology and Oceanography, 2005, 50(1): 113-127.

[8] Glud RN, Tengberg A, Kühl M et al. An in situ instrument for planar $\mathrm{O}_{2}$ optode measurements at benthic interfaces. Limnology and Oceanography, 2001, 46(8): 2073-2080.

[9] Klimant I, Kühl M, Glud RN et al. Optical measurement of oxygen and temperature in microscale: strategies and biological applications. Sensors \& Actuators: B. Chemical, 1997, 38(1-3): 29-37.

[10] Holst G, Kohls O, Klimant I et al. A modular luminescence lifetime imaging system for mapping oxygen distribution in biological samples. Sensors \& Actuators B: Chemical, 1998, 51(1-3): 163-170.

[11] Berg P, Risgaard-Petersen N, Rysgaard S. Interpretation of measured concentration profiles in sediment pore water. Limnology and Oceanography, 1998, 43(7): 1500-1510.

[12] Berg P, Røy H, Janssen F et al. Oxygen uptake by aquatic sediments measured with a novel non-invasive eddy-correlation technique. Marine Ecology Progress Series, 2003, 261: 75-83.

[13] Kuwae T, Kamio K, Inoue T et al. Oxygen exchange flux between sediment and water in an intertidal sandflat, measured in situ by the eddy-correlation method. Marine Ecology Progress Series, 2006, 307: 59-68.

[14] Polerecky L, Franke U, Werner U et al. High spatial resolution measurement of oxygen consumption rates in permeable sediments. Limnology and Oceanography Methods, 2005, 3: 75-85.

[15] Otubu JE, Hunter JV, Francisco KL et al. Temperature effects on Tubificid worms and their relation to sediment oxygen demand. Journal of Environmental Science and Health, Part A, 2006, 41(8): 1607-1613.

[16] Coles SL, Jokiel PL. Effects of temperature on photosynthesis and respiration in hermatypic corals. Marine Biology, 1977, 43(3): 209-216. 
[17] Hancke K, Glud RN. Temperature effects on respiration and photosynthesis in three diatom-dominated benthic communities. Aquatic Microbial Ecology, 2004, 37(3): 265-281.

[18] Dedieu K, Rabouille C, Thouzeau G et al. Benthic $\mathrm{O}_{2}$ distribution and dynamics in a Mediterranean lagoon (Thau, France): An in situ microelectrode study. Estuarine, Coastal and Shelf Science, 2007, 72(3): 393-405

[19] Andersson E, Brunberg AK. Net autotrophy in an oligotrophic lake rich in dissolved organic carbon and with high benthic primary production. Aquatic Microbial Ecology, 2006, 43(1): 1-10.

[20] Jørgensen BB, Glud RN, Holby O. Oxygen distribution and bioirrigation in Arctic fjord sediments (Svalbard, Barents Sea). Marine Ecology Progress Series, 2005, 292: 85-95.

[21] Lohrer AM, Thrush SF, Gibbs MM. Bioturbators enhance ecosystem function through complex biogeochemical interactions. Nature, 2004, 431(7012): 1092-1095.

[22] Aller RC. Benthic fauna and biogeochemical processes in marine sediments: the role of burrow structures. John Wiley and Sons Ltd, 1988.

[23] Forster S, Graf G. Impact of irrigation on oxygen flux into the sediment: intermittent pumping by Callianassa subterranea and "piston-pumping" by Lanice conchilega. Marine Biology, 1995, 123(2): 335-346.

[24] Kim KH, Kim D. Seasonal and spatial variability of sediment oxygen fluxes in the Beobsan intertidal flat of Taean Bay, mid-western Korean Peninsula. Geosciences Journal, 2007, 11(4): 323-329.

[25] Banse K. Mass-scaled rates of respiration and intrinsic growth in very small invertebrates. Marine Ecology Progress Series, 1982, 9(3): 281-297

[26] Archer D, Devol A. Benthic oxygen fluxes on the Washington shelf and slope: A comparison of in situ microelectrode and chamber flux measurements. Limnology and Oceanography, 1992, 37(3): 614-629.

[27] Jørgensen BB, Revsbech NP. Diffusive boundary layers and the oxygen uptake of sediments and detritus. Limnology and Oceanography, 1985, 30(1): 111-122.

[28] Qu W, Morrison RJ, West RJ. Inorganic nutrient and oxygen fluxes across the sediment-water interface in the inshore macrophyte areas of a shallow estuary (Lake Illawarra, Australia). Hydrobiologia, 2003, 492(1): 119-127.

[29] Risgaard-Petersen N, Rysgaard S, Nielsen LP et al. Diurnal variation of denitrification and nitrification in sediments colonized by benthic microphytes. Limnology and Oceanography, 1994, 39(3): 573-579.

[30] Reimers CE, Smith Jr KL. Reconciling measured and predicted fluxes of oxygen across the deep sea sediment-water interface. Limnology and Oceanography, 1986, 31(2): 305-318.

[31] Gelda RK, Auer MT, Effler SW. Determination of sediment oxygen demand by direct measurement and by inference from reduced species accumulation. Marine and Freshwater Research, 1995, 46: 81-88.

[32] Revsbech NP, Jørgensen BB. Microelectrodes: their use in microbial ecology. Advances in Microbial Ecology, 1986, 9: 293-352.

[33] Roy H, Huttel M, Jørgensen BB. The role of small-scale sediment topography for oxygen flux across the diffusive boundary layer. Limnology and Oceanography, 2002, 47(3): 837-847.

[34] Andersson E, Helder W. Comparison of oxygen microgradients, oxygen flux rates and electron transport system activity in coastal marine sediments. Marine Ecology Progress Series, 1987, 37: 259-264.

[35] Grenz C, Denis L, Boucher G et al. Spatial variability in sediment oxygen consumption under winter conditions in a lagoonal system in New Caledonia (South Pacific). Journal of Experimental Marine Biology and Ecology, 2003, 285: 33-47.

[36] Rabouille C, Denis L, Dedieu K et al. Oxygen demand in coastal marine sediments: comparing in situ microelectrodes and laboratory core incubations. Journal of Experimental Marine Biology and Ecology, 2003, 285-286: 49-69.

[37] Fenchel T, Glud RN. Benthic primary production and $\mathrm{O}_{2}-\mathrm{CO}_{2}$ dynamics in a shallow water sediment: Spatial and temporal heterogeneity. Ophelia, 2000, 53(3): 159-171. 